\title{
Calibration Results for Non-Expected Utility Theories*
}

\author{
Zvi Safra ${ }^{\dagger}$ and Uzi Segal ${ }^{\ddagger}$
}

July 24, 2006

\begin{abstract}
Rabin [17] proved that a low level of risk aversion with respect to small gambles leads to a high, and absurd, level of risk aversion with respect to large gambles. Rabin's arguments strongly depend on expected utility theory, but we show that similar arguments apply to many non-expected utility theories.
\end{abstract}

\section{Introduction}

One of the fundamental hypotheses about decision makers' behavior in risky environments is that they evaluate actions by considering possible final wealth levels. Throughout the last fifty years the final-wealth hypothesis has been widely used in the classical theory of expected utility as well as in its applications. Moreover, many of the new alternatives to expected utility, alternatives that were developed during the last twenty five years in order to overcome the limited descriptive power of expected utility, are also based on the hypothesis that only final wealth levels matter.

*We thank Eddie Dekel, Larry Epstein, Simon Grant, Eran Hanany, Edi Karni, Mark Machina, Matthew Rabin, Ariel Rubinstein, and Shunming Zhang for their suggestions. Zvi Safra thanks the Israel Institute of Business research for its financial support.

${ }^{\dagger}$ Faculty of Management, Tel Aviv University, Tel Aviv 69978, Israel and the Academic College of Tel Aviv Yaffo (safraz@post.tau.ac.il).

$\ddagger$ Dept. of Economics, Boston College, Chestnut Hill MA 02467, USA (segalu@bc. edu). 
The final-wealth hypothesis is analytically tractable as it assumes that decision makers behave according to a unique, universal preference relation over final-wealth distributions. Suggested deviations from this hypothesis require much more elaborate and complex analysis. For example, postulating that decision makers ignore final wealth levels and, instead, care about possible gains and losses may require using many preference relations and necessitates the need for a mechanism that defines the appropriate reference points. However, the poor descriptive power of some of the final-wealth models and, in particular, of the final-wealth expected utility models, have increased the popularity of gain-losses model such as prospect theory and its offsprings.

Recently, Rabin [17] offered a strong theoretical argument against finalwealth expected utility theory: Seemingly innocuous levels of risk aversion with respect to small gambles lead to enormous levels of risk aversion with respect to large gambles. For example, decision makers who at all wealth levels beneath $\$ 300,000$ reject an even chance of winning $\$ 110$ or losing $\$ 100$ will also reject at the wealth level of $\$ 290,000$ an even chance of loosing $\$ 2000$ and gaining $\$ 12,000,000 .^{1}$

A possible response to Rabin's argument is that its basic assumption is actually wrong: No reasonable expected utility decision maker will reject the small gamble at such a wide range. Palacios-Huerta and Serrano [15] claim that even if expected utility decision makers are risk averse, moderate observed levels of relative risk aversion imply that absolute risk aversion must go down to zero as wealth increases. Hence, at sufficiently large wealth levels, decision makers will accept the small (but positive expected value) gamble (see also LeRoy [12]).

Our analysis rejects this defence of expected utility theory. As we show in section 2, Rabin's calibration results can be strengthened by restricting the length of the intervals to less than forty thousands. Over such intervals, the claim of Palacios-Huerta and Serrano is less compelling.

A natural conclusion from Rabin's argument and from the results of section 2 is that final-wealth expected utility should be replaced with more general final-wealth theories. Indeed, it can easily be seen that rank-dependent with linear utility (Yaari [22]) is capable of exhibiting both a relatively strong

\footnotetext{
${ }^{1}$ For an earlier claim that a low level of risk aversion in the small implies huge risk aversion at the large, although without detailed numerical estimates, see Hansson [11] and Epstein [9].
} 
aversion to small gambles and a sensible degree of risk aversion with respect to large gambles. Nevertheless, our main result is that simple extensions of Rabin's argument apply to many known models of final-wealth non-expected utility theories, including Chew's [3] weighted utility, betweenness (Dekel [8], Chew [3]), some forms of quadratic utility (Machina [13], Chew, Epstein, and Segal [5]), and some versions of the more general differentiable non-expected utility model (Machina [13]). We prove our calibration results by assuming some restrictions on these functionals, requiring them to satisfy forms of monotonicity regarding risk aversion. This is done by utilizing Machina's [13] hypotheses 1 and 2. We use these methods to obtain the calibration results of section 4 below.

Not all models are covered by our analysis, which crucially depends on the differentiability of the local utility functions approximating the general functionals. We deal with the nondifferential case in another paper [18].

\section{Calibration Results for Expected Utility}

Rabin's [17] results are directed at showing that rejections of small, favorable, even bets must lead to the rejection of enormously favorable even bets. In this section we first show that Rabin's results hold even if the range at which the small lotteries are rejected is significantly smaller than the one suggested by Rabin. We then show that rejections of small even bets must also lead to the rejection of extremely profitable low risk investment opportunities.

Following Rabin, consider a risk averse expected utility maximizer with a concave vNM utility function $u$, who, for $\ell<g$, is rejecting the lottery $\left(-\ell, \frac{1}{2} ; g, \frac{1}{2}\right)$ at all wealth levels $x$ in a given interval $[a, b] .{ }^{2}$ We assume, for simplicity, that $b-a=k(\ell+g)$ for some integer $k$.

Rejecting the lottery $\left(-\ell, \frac{1}{2} ; g, \frac{1}{2}\right)$ at $a+\ell$ implies $u(a+\ell)>\frac{1}{2}[u(a)+$ $u(a+\ell+g)]$, hence $u(a+\ell)-u(a)>u(a+\ell+g)-u(a+\ell)$. By concavity, $u^{\prime}(a) \geqslant[u(a+\ell)-u(a)] / \ell$ and

$$
u^{\prime}(a+\ell+g) \leqslant \frac{u(a+\ell+g)-u(a+\ell)}{g}<\frac{u(a+\ell)-u(a)}{\ell} \frac{\ell}{g} \leqslant \frac{\ell}{g} u^{\prime}(a)
$$

Similarly, as $b=a+k(\ell+g)$ and $k=\frac{b-a}{\ell+g}$, we get

\footnotetext{
${ }^{2}$ The probabilities need not be the same. This analysis is also valid for decision makers who, for example, reject lotteries of the form $(-80,0.1 ; 10,0.9)$.
} 


$$
u^{\prime}(b)<u^{\prime}(a)\left(\frac{\ell}{g}\right)^{\frac{b-a}{\ell+g}}
$$

Concavity implies that for every $c, u(c+\ell+g) \leqslant u(c)+(\ell+g) u^{\prime}(c)$, hence

$$
u(b) \leqslant u(a)+(\ell+g) u^{\prime}(a) \sum_{i=1}^{\frac{b-a}{\ell+g}}\left(\frac{\ell}{g}\right)^{i-1}
$$

Likewise, for every $c, u(c-\ell-g) \leqslant u(c)-(\ell+g) u^{\prime}(c)$, hence

$$
u(a) \leqslant u(b)-(\ell+g) u^{\prime}(b) \sum_{i=1}^{\frac{b-a}{\ell+g}}\left(\frac{g}{\ell}\right)^{i-1}
$$

Normalizing $u(a)=0$ and $u^{\prime}(a)=1$ we obtain from eqs. (1) and (2)

$$
u^{\prime}(b) \leqslant\left(\frac{\ell}{g}\right)^{\frac{b-a}{\ell+g}} \text { and } u(b) \leqslant(\ell+g) \frac{1-\left(\frac{\ell}{g}\right)^{\frac{b-a}{\ell+g}}}{1-\frac{\ell}{g}}
$$

For concave $u$ we now obtain that for every $x \notin[a, b]$

$$
u(x) \leqslant \begin{cases}-(a-x) & x<a \\ u(b)+(x-b)\left(\frac{\ell}{g}\right)^{\frac{b-a}{\ell+g}} & x>b\end{cases}
$$

Alternatively, a normalization with $u(b)=0$ and $u^{\prime}(b)=1$ gives (by eqs. (1) and (3))

$$
u^{\prime}(a) \geqslant\left(\frac{g}{\ell}\right)^{\frac{b-a}{\ell+g}} \text { and } u(a) \leqslant-(\ell+g) \frac{1-\left(\frac{g}{\ell}\right)^{\frac{b-a}{\ell+g}}}{1-\frac{g}{\ell}}
$$

and hence, for every $x \notin[a, b]$

$$
u(x) \leqslant \begin{cases}u(a)-(a-x)\left(\frac{g}{\ell}\right)^{\frac{b-a}{\ell+g}} & x<a \\ x-b & x>b\end{cases}
$$


Inequalities (4) and (5) imply that if for all wealth levels $x$ between $a$ and $b$ the decision maker rejects the lottery $\left(-\ell, \frac{1}{2} ; g, \frac{1}{2}\right)$, then when his wealth level is $a$, he will also reject any lottery of the form $(-L, p ; G, 1-p), G>b-a$, provided that

$$
L>\left[(\ell+g) \frac{1-\left(\frac{\ell}{g}\right)^{\frac{b-a}{\ell+g}}}{1-\frac{\ell}{g}}+(G+a-b)\left(\frac{\ell}{g}\right)^{\frac{b-a}{\ell+g}}\right] \frac{1-p}{p}
$$

Table 1 offers the value of $L$ for different levels of $G, b-a$, and $g$ when $\ell=100$ and $p=\frac{1}{2}$. If $p \neq \frac{1}{2}$, the values should be multiplied by $\frac{1-p}{p}$.

\begin{tabular}{|cc||c|c|c|c|}
\hline$G$ & $b-a$ & $g=101$ & $g=105$ & $g=110$ & $g=125$ \\
\hline \hline 200,000 & 20,000 & 79,637 & 5,810 & 2,330 & 1,125 \\
& 40,000 & 39,586 & 4,316 & 2,310 & 1,125 \\
\hline $1,000,000$ & 20,000 & 376,873 & 12,662 & 2,421 & 1,125 \\
& 40,000 & 150,023 & 4,375 & 2,310 & 1,125 \\
\hline $10,000,000$ & 20,000 & $3,720,787$ & 89,752 & 3,450 & 1,125 \\
& 40,000 & $1,392,440$ & 5,035 & 2,310 & 1,125 \\
\hline
\end{tabular}

Table 1: If the decision maker rejects $\left(-100, \frac{1}{2} ; g, \frac{1}{2}\right)$ at all wealth levels between $a$ and $b$, then at $a$ he also rejects $\left(-L, \frac{1}{2} ; G, \frac{1}{2}\right)$, values of $L$ entered in the table.

For example, if the decision maker rejects $\left(-100, \frac{1}{2} ; 110, \frac{1}{2}\right)$ on a range of 20,000 , then he also rejects the lottery $\left(-3,450, \frac{1}{2} ; 10,000,000, \frac{1}{2}\right)$. Multiplying by $\frac{1-p}{p}$ for $1-p=\frac{1}{100,000}$, we obtain that this decision maker will refuse to pay even four cents for a 1:100,000 chance of winning 10 million dollars!

Inequalities (6) and (7) imply that if for all wealth levels $x$ between $a$ and $b$ the decision maker rejects the lottery $\left(-\ell, \frac{1}{2} ; g, \frac{1}{2}\right)$, then he will also reject, at $b$, any lottery of the form $(-(b-a), p ; G, 1-p)$, provided that

$$
p(\ell+g) \frac{\left(\frac{g}{\ell}\right)^{\frac{b-a}{\ell+g}}-1}{\frac{g}{\ell}-1}>(1-p) G \Longleftrightarrow p>G /\left[(\ell+g) \frac{\left(\frac{g}{\ell}\right)^{\frac{b-a}{\ell+g}}-1}{\frac{g}{\ell}-1}+G\right]
$$

In Table $2, \ell=100$ and the wealth level is $b$. The table presents, for different combinations of $L=b-a, G$, and $g$, values of $p$ such that a rejection of $\left(-100, \frac{1}{2} ; g, \frac{1}{2}\right)$ at all $x \in[b-L, b]$ leads to a rejection of $(-L, p ; G, 1-p)$ at $b$. 


\begin{tabular}{|cc||c|c|c|c|}
\hline$L$ & $G$ & $g=101$ & $g=105$ & $g=110$ & $g=125$ \\
\hline \hline \multirow{2}{*}{20,000} & 100,000 & 0.7462 & 0.1740 & 0.0054 & $2.7 \cdot 10^{-7}$ \\
& $1,000,000$ & 0.9671 & 0.6781 & 0.0516 & $2.7 \cdot 10^{-6}$ \\
& $100,000,000$ & 0.9996 & 0.9952 & 0.8447 & $2.7 \cdot 10^{-4}$ \\
\hline \multirow{3}{*}{30,000} & 100,000 & 0.5929 & 0.0189 & $5.8 \cdot 10^{-5}$ & $1.3 \cdot 10^{-11}$ \\
& $1,000,000$ & 0.9357 & 0.1621 & $5.8 \cdot 10^{-4}$ & $1.3 \cdot 10^{-10}$ \\
& $100,000,000$ & 0.9993 & 0.9508 & 0.0550 & $1.3 \cdot 10^{-8}$ \\
\hline \multirow{3}{*}{50,000} & 100,000 & 0.3137 & $1.7 \cdot 10^{-4}$ & $6.6 \cdot 10^{-9}$ & $3.2 \cdot 10^{-20}$ \\
& $1,000,000$ & 0.8205 & 0.0017 & $6.6 \cdot 10^{-8}$ & $3.2 \cdot 10^{-19}$ \\
& $100,000,000$ & 0.9978 & 0.1421 & $6.6 \cdot 10^{-6}$ & $3.2 \cdot 10^{-17}$ \\
\hline
\end{tabular}

Table 2: If the decision maker rejects $\left(-100, \frac{1}{2} ; g, \frac{1}{2}\right)$ at all wealth levels in $[b-L, b]$, then at $b$ he also rejects $(-L, p ; G, 1-p)$, values of $p$ entered in the Table.

We believe that the values of Table 2 are even more disturbing than those of Rabin [17], as many of the entries represent an almost sure gain of huge amounts of money, where with a very small probability less than 50,000 may be lost. For example, if the decision maker rejects $\left(-100, \frac{1}{2} ; 110, \frac{1}{2}\right)$ on a range of 30,000 , then he also rejects the lottery $\left(-30,000, \frac{1}{1700} ; 1,000,000, \frac{1699}{1700}\right)$. In the next section we show that similar tables can be constructed for many non-expected utility models.

\section{Definitions and Assumptions}

Having Tables 1 and 2 in mind, we begin with the following definition.

Definition 1 The vector $(\ell, g, L, G, c)$ is an upper calibration quintuple if a risk averse expected utility decision maker who is rejecting $\left(-\ell, \frac{1}{2} ; g, \frac{1}{2}\right)$ at all wealth levels in $[w, w+c]$ will also reject $\left(-L, \frac{1}{2} ; G, \frac{1}{2}\right)$ at the wealth level $w$, for all $w$. The vector $(\ell, g, L, G, \varepsilon)$ is a lower calibration quintuple if a risk averse expected utility decision maker who is rejecting $\left(-\ell, \frac{1}{2} ; g, \frac{1}{2}\right)$ at all wealth levels in $[w-L, w]$ will also reject $(-L, \varepsilon ; G, 1-\varepsilon)$ at the wealth level $w$, for all $w$.

We assume throughout that preferences over distributions are representable by functionals $V$ that are risk averse with respect to mean-preserving spreads, monotonically increasing with respect to first order stochastic dominance, continuous with respect to the topology of weak convergence, and 
that they display a modest degree of smoothness in the sense that at every distribution $F$, there is a hyperplane containing all the tangent lines at $F$ to the indifference curve of $V$ through $F$. Denote the set of all such functionals by $\mathcal{V}$.

Observe that the aforementioned hyperplane is an indifference set of an expected utility functional with a unique (up to positive affine transformations) vNM utility function $u(\cdot ; F)$, which is called the local utility at $F$ (see Machina [13]). ${ }^{3}$ According to the context, utility functionals are defined over lotteries (of the form $X=\left(x_{1}, p_{1} ; \ldots ; x_{n}, p_{n}\right)$ ) or over cumulative distribution functions (denoted $F, H$ ). Degenerate cumulative distribution functions are denoted $\delta_{x}$. Machina [13] introduced the following definitions.

Definition 2 (a) The functional $V \in \mathcal{V}$ satisfies Hypothesis 1 (H1) if for every distribution $F,-\frac{u^{\prime \prime}(x ; F)}{u^{\prime}(x ; F)}$ is a nonincreasing function of $x$.

(b) The functional $V \in \mathcal{V}$ satisfies Hypothesis 2 (H2) if for all $F$ and $H$ such that $F$ dominates $H$ by first order stochastic dominance and for all $x$

$$
-\frac{u^{\prime \prime}(x ; F)}{u^{\prime}(x ; F)} \geqslant-\frac{u^{\prime \prime}(x ; H)}{u^{\prime}(x ; H)}
$$

In the sequel, we will also use the opposite of these assumptions, denoted $\neg \mathrm{H} 1$ and $\neg \mathrm{H} 2$. $\neg \mathrm{H} 1$ says that for every distribution $F,-\frac{u^{\prime \prime}(x ; F)}{u^{\prime}(x ; F)}$ is a nondecreasing function of $x$ while $\neg \mathrm{H} 2$ says that if $F$ dominates $H$ by first order stochastic dominance, then for all $x,-\frac{u^{\prime \prime}(x ; F)}{u^{\prime}(x ; F)} \leqslant-\frac{u^{\prime \prime}(x ; H)}{u^{\prime}(x ; H)}$. We say that H1 (or $\neg \mathrm{H} 1$ ) is satisfied in $\mathcal{S}$ if it is satisfied at all $F \in \mathcal{S}$. Similarly, H2 (or $\neg \mathrm{H} 2)$ is satisfied in $\mathcal{S}$ if it applies to all $F, H \in \mathcal{S}$.

By definition, $\mathrm{H} 1$ and $\mathrm{H} 2$ need differentiability of the local utility functions. This is not a trivial assumption, and it is closely associated with orders of risk aversion. ${ }^{4}$ We discuss below models that are compatible with these assumptions.

Machina $[13,14]$ shows that $\mathrm{H} 1$ and $\mathrm{H} 2$ conform with many violations of expected utility like the Allais paradox, the common ratio effect [1], and

\footnotetext{
${ }^{3}$ By Dekel [8], the existence of such hyperplanes does not require Fréchet differentiability (as in Machina [13]) or even Gâteaux differentiability (as in Chew, Karni, and Safra $[6])$.

${ }^{4} V$ represents first [second] order risk aversion if the risk premium the decision maker is willing to pay to avoid playing $t \cdot X \equiv\left(t x_{1}, p_{1} ; \ldots ; t x_{n}, p_{n}\right)$ for $\mathrm{E}[X]=0$ converges to zero at the same rate as $t\left[t^{2}\right]$. See Segal and Spivak $[19,20]$.
} 
the mutual purchase of insurance policies and lottery tickets. Hypothesis 2 implies that for given $x>y>z$, indifference curves in the set $\{(x, p ; y, 1$ $-p-q ; z, q): p+q \leqslant 1\}$ become steeper as one moves from $\delta_{z}$ to $\delta_{x}$. The experimental evidence concerning $\mathrm{H} 2$ is inconclusive. Battalio, Kagel, and Jiranyakul [2] and Conlisk [7] suggest that indifference curves become less steep as one moves closer to either $\delta_{x}$ or $\delta_{z}$. But Conlisk's experiment does not prove a violation of $\mathrm{H} 2$ near $\delta_{x}$. In this part of the experiment, subjects were asked to rank $B=(5,0.88 ; 1,0.11 ; 0,0.01)$ and $B^{*}=(5,0.98 ; 0,0.02)$. Most ranked $B^{*}$ higher than $B$. But this pair does not dominate the pair $(1,1)$ and $(5,0.1 ; 1,0.89 ; 0,0.01)$ of the Allais paradox, and therefore the fact that most subjects prefer $(1,1)$ to $(5,0.1 ; 1,0.89 ; 0,0.01)$ does not prove a violation of H2. Moreover, we suspect that most subjects would prefer $(5,0.89 ; 1,0.11)$ to $(5,0.99 ; 0,0.01)$, which is consistent with $\mathrm{H} 2$. Battalio et al. did find some violations of this assumption, but as most of their subjects were consistent with expected utility theory, only a small minority of them violated this hypothesis. For further citations of violations of H2, see Starmer [21, Sec. 5.1.1].

\section{Calibration Results for Non EU Preferences}

We start by analyzing the set of quasi concave functionals, that is, functionals where $V(F) \geqslant V(H)$ implies for all $\alpha \in(0,1), V(\alpha F+(1-\alpha) H) \geqslant V(H)$. An important subset of this is the set of betweenness functionals (Dekel [8], Chew [4]), where indifference sets are hyperplanes: If $F$ and $H$ are in the indifference set $\mathcal{I}$, then for all $\alpha \in(0,1)$, so is $\alpha F+(1-\alpha) H$. Formally: $V$ satisfies betweenness if for all $F$ and $H$ satisfying $V(F) \geqslant V(H)$ and for all $\alpha \in(0,1), V(F) \geqslant V(\alpha F+(1-\alpha) H) \geqslant V(H)$.

Theorem 1 Let $V \in \mathcal{V}$ be a quasi concave functional and let $(\ell, g, L, G, \varepsilon)$ and $(\ell, g, \bar{L}, \bar{G}, c)$ be lower and upper calibration quintuples, respectively. Define $\mathcal{S}_{1}=\{X: \operatorname{supp}(X) \subseteq[a, b]\}$ for some $a$ and $b$ satisfying $b-a=\max \{L+$ $g, c+\ell\}$ and assume that, for all $x \in[a, b], V(x, 1)>V\left(x-\ell, \frac{1}{2} ; x+g, \frac{1}{2}\right)$.

1. If $V$ satisfies $\mathrm{H} 1$ or $\mathrm{H} 2$ on $\mathcal{S}_{1}$ then $V(b, 1)>V(b-g-L, \varepsilon ; b-g+G, 1-\varepsilon)$.

2. If $V$ satisfies $\neg \mathrm{H} 1$ or $\neg \mathrm{H} 2$ on $\mathcal{S}_{1}$ then $V(a, 1)>V\left(a-\bar{L}, \frac{3}{4} ; a+\bar{G}, \frac{1}{4}\right)$.

In other words, adjustments not larger than $g$ are made in the outcomes of the large lotteries of Table 2 and the probabilities of Table 1 are changed to 
$\frac{3}{4}$ and $\frac{1}{4}$. Obviously, the rejection of the new big lotteries is still absurd. ${ }^{5}$ If we assume betweenness, we can prove that rejected large lotteries are exactly those given in Tables 1 and 2 (see [18]).

Quadratic utility (Machina [13], Chew, Epstein, and Segal [5]) is given by $V(F)=\iint \varphi(x, y) d F(x) d F(y)$ for some symmetric, continuous, and monotonic function $\varphi$. If one indifference of a quadratic function is quasi concave, then so are all indifference curves above it. ${ }^{6}$ The local utility of quadratic functionals is given by $u(x ; F)=\int \varphi(x, y) d F(y)$. It is easy to verify that H2 $[\neg \mathrm{H} 2]$ is satisfied if $\varphi_{x x y} \varphi_{x}-\varphi_{x x} \varphi_{x y}<[>] 0$ and that H1 [ $\left.\neg \mathrm{H} 1\right]$ is satisfied if $\varphi_{x x x} \varphi_{x}-\varphi_{x x}^{2}>[<] 0$.

Chew's [3] weighted utility is given by $V(F)=\int v d F / \int h d F$ with the local utility $u\left(x ; \delta_{w}\right)=v(x)-\frac{v(w)}{h(w)} h(x)$. This function induces either fanning out or fanning in indifference curves on probability triangles $\{(x, p ; y, 1-$ $p-q ; z, q)\}$ for given $x>y>z$, but in general it satisfies $\mathrm{H} 2[\neg \mathrm{H} 2]$ whenever $\left[v^{\prime \prime}(x) h^{\prime}(x)-v^{\prime}(x) h^{\prime \prime}(x)\right]\left[v^{\prime}(w) h(w)-v(w) h^{\prime}(w)\right]>[<] 0$. Monotonicity with respect to first order stochastic dominance implies that $v(w) h^{\prime}(w)-$ $v^{\prime}(w) h(w)$ does not change sign [3, Corollary 5]. ${ }^{7} \mathrm{H} 2$ and $\neg \mathrm{H} 2$ thus require $\left[v^{\prime \prime}(x) h^{\prime}(x)-v^{\prime}(x) h^{\prime \prime}(x)\right]$ not to change its sign. Although not all weighted utility functionals satisfy these assumptions, we can prove directly that the results of Theorem 1 apply to this functional without making any assumptions like $\mathrm{H} 1$ or $\mathrm{H} 2$ (see [18]).

The proof of the theorem utilizes the local structure of the given functionals. The rejection of the small lottery $\left(-\ell, \frac{1}{2} ; g, \frac{1}{2}\right)$ at $b$ implies, by quasi concavity, that the local utility function $u^{*}$ at $\left(b-\ell, \frac{1}{2} ; b+g, \frac{1}{2}\right)$ prefers $(b, 1)$ to the lottery $\left(b-\ell, \frac{1}{2} ; b+g, \frac{1}{2}\right)$. If $\mathrm{H} 1$ is satisfied, then $u^{*}$ also prefers $(x, 1)$ to the lottery $\left(x-\ell, \frac{1}{2} ; x+g, \frac{1}{2}\right)$, for all $x \leqslant b$. Table 2 then implies that $u^{*}$ rejects large and attractive lotteries. We use this and quasi-concavity to show that the original functional rejects these large lotteries as well. Alternatively, assume H2. For all $x \in[a, b-g]$, the decision maker prefers $(x, 1)$ to $\left(x-\ell, \frac{1}{2} ; x+g, \frac{1}{2}\right)$, hence, as above, the local utility function at $\left(x-\ell, \frac{1}{2} ; x+g, \frac{1}{2}\right)$ prefers $(x, 1)$ to the lottery $\left(x-\ell, \frac{1}{2} ; x+g, \frac{1}{2}\right)$. By H2, so does the local utility at $(b, 1)$ and the argument follows as above. A formal

\footnotetext{
${ }^{5}$ Assuming $\neg \mathrm{H} 2$ we actually prove that $V(a, 1)>V\left(a-\bar{L}, \frac{1}{2}+\frac{\ell}{L} ; a+\bar{G}, \frac{1}{2}-\frac{\ell}{L}\right)$. See eq. (14) at the end of the proof of Theorem 1.

${ }^{6}$ Conditions implying quasi concavity are discussed in [5, fnt. 6].

${ }^{7}$ In Chew's [3] notation, $V(F)=\int \alpha \phi d F / \int \alpha d F$. To obtain our representation, let $h=\alpha$ and $\phi=v / h$. Monotonicity in $x$ of $\alpha(x)(\phi(x)-\phi(s))$ for all $s$ is equivalent to monotonicity of $v(x)-h(x) v(s) / h(s)$, that is, to $v^{\prime}(x)-h^{\prime}(x) v(s) / h(s) \neq 0$.
} 
proof of these and the other cases appears in the Appendix.

The following example shows the necessity of the $\mathrm{H}$ assumptions.

Example 1 Let $u_{w}(x)=\min \left\{x, \frac{w+x}{2}\right\}$ and define $V(F)$ to be that number $w$ such that $\mathrm{E}\left[u_{w}(F)\right]=w$. This is a special case of Gul's [10] disappointment aversion theory, which satisfies betweenness. Its local utilities are $u_{w}$ which are nondifferentiable, and hence do not satisfy any of the four hypotheses. Clearly these preferences reject $\left(-100, \frac{1}{2} ; g, \frac{1}{2}\right)$ for all $g<200$, but accept $\left(-L, \frac{1}{2} ; G, \frac{1}{2}\right)$ for all $G>2 L$.

Next we analyze general preferences that are not necessarily quasi concave. To make sure that they are sufficiently smooth we assume Gâteaux differentiability (see Zeidler [23, p. 191]). If the functional $V$ is Gâteaux differentiable at $F$, then there exists a function $u(\cdot ; F)$ such that for every $H$ and $t$,

$$
V((1-t) F+t H)-V(F)=t \int u(x ; F) d(H-F)(x)+o(t)
$$

Although all indifference sets of betweenness functionals are hyperplanes, these functionals are not necessarily Gâteaux differentiable (see Dekel [8]).

The following lemma is needed since the calibration results for expected utility rely on the concavity of the vNM function. It extends Machina's [13] result for Fréchet differentiable functionals to the class of all Gâteaux differentiable functionals.

Lemma 1 All local utilities of a Gâteaux differentiable $V \in \mathcal{V}$ are concave.

The next result relates to general (not necessarily quasi concave) Gâteaux differentiable functions. Unlike the first theorem, it yields only a subset of lower calibration quintuples.

Suppose that for all $x \in[a, b], V(x, 1)>V\left(x-\ell, \frac{1}{2} ; x+g, \frac{1}{2}\right)$. By the continuity of $V$, there exists $\hat{\varepsilon}>0$ such that $\forall \varepsilon \in(0, \hat{\varepsilon})$ and $\forall x \in[a, b]$, $V(a, \varepsilon ; x, 1-\varepsilon)>V\left(a, \varepsilon ; x-\ell, \frac{1-\varepsilon}{2} ; x+g, \frac{1+\varepsilon}{2}\right)$. In theorem 2 we therefore assume directly the existence of such $\hat{\varepsilon}$.

Theorem 2 Let $V \in \mathcal{V}$ be a Gâteaux differentiable functional. Let $\ell, g, L \in$ $\Re$, let $b-a=L+g$, and let $\hat{\varepsilon}$ be as above. Define $\mathcal{S}_{2}=\{X: \operatorname{Supp} X \subset[a, b]\}$. Let $\varepsilon<\hat{\varepsilon}$ and let

$$
G<\frac{\varepsilon}{1-\varepsilon}(\ell+g) \frac{\left(\frac{g}{\ell}\right)^{\frac{b-a}{\ell+g}}-1}{\frac{g}{\ell}-1}
$$


If $V$ satisfies H2 on $\mathcal{S}_{2}$, then $V(b, 1) \geqslant V(b-g-L, \varepsilon ; b-g+G, 1-\varepsilon)$.

For example, let $a=b-40 K$. If for all $x \in[a, b]$ the decision maker prefers $(a, 0.002 ; x, 0.998)$ to $(a, 0.002 ; x-100,0.499 ; x+110,0.499)$, then he also prefers $(b, 1)$ to $\left(b-40 K, 0.002 ; 3.4 \cdot 10^{8}, 0.998\right)$. Gâteaux differentiable quadratic utility is an example for a functional covered by this theorem.

The first part of the proof of Theorem 2 is similar to that of Theorem 1 . The rejection of the small lottery $\left(-\ell, \frac{1}{2} ; g, \frac{1}{2}\right)$ at all $x \in[a, b-g]$ implies, by differentiability, that at some distribution on the line segment connecting $(x, 1)$ and $\left(x-\ell, \frac{1}{2} ; x+g, \frac{1}{2}\right)$, the local utility function $\bar{u}_{x}$ prefers $(x, 1)$ to the lottery $\left(x-\ell, \frac{1}{2} ; x+g, \frac{1}{2}\right)$. By H2, so does the local utility $\bar{u}$ at $(b, 1)$. Hence, $\bar{u}$ rejects some of the attractive large lotteries of Table 2 . Next we show that a similar property holds along the line segment connecting $(b, 1)$ and $(b-g-L, \varepsilon: b-g+G, 1-\varepsilon)$. By Gâteaux differentiability, the decision maker rejects the large lotteries as well.

The requirement that $V$ rejects mean preserving spreads is essential. Machina [13, Sec. 4.5] provides an example for a functional $V$ that satisfies H2, rejects symmetric lotteries, and yet does not reject attractive large lotteries. This functional does not reject all mean preserving spreads, and indeed, its local utility functions are not globally concave.

\section{Discussion}

Rabin [17] proved that if a risk averse expected utility maximizer is modestly risk averse in the small, then he must be absurdly risk averse in the large. This paper shows that this criticism is not limited to expected utility theory - similar arguments can be made against many of its known transitive alternatives. For these extensions we need to assume H1 or H2 (or their opposites), that relate to changes in attitudes towards risk that result from moving from one lottery to another.

Both $\mathrm{H} 1$ and $\mathrm{H} 2$ assume differentiable local utilities, hence they rule out first order risk aversion (see $[19,20]$ ). To this class belong disappointment aversion (Gul [10]) and rank dependent utilities (Quiggin [16]). To obtain calibration results for such models we need an alternative tool, which is based on the rejection of small lotteries at the face of background risk (see Safra and Segal [18]).

Rabin's original arguments seem to be relevant only for expected utility theory. Essentially, these arguments tie together small degree of concavity for 
small neighborhoods at many points to obtain a lot of concavity with respect to big lotteries. This can be done since expected utility theory permits only one function. In this paper we show that this analysis does not require linearity in the probabilities, and can be extended to situations where more than one function is used (like Chew's [3] weighted utility), provided some structure is assumed regarding the decision maker's attitude towards risk when one moves from one distribution to another.

This paper provides sufficient, not necessary, conditions for the existence of calibration results. Other results are possible - for example, we can prove Theorem 1 for general Gâteaux differentiable functionals satisfying either $\mathrm{H} 1$ and $\neg \mathrm{H} 2$, or $\neg \mathrm{H} 1$ and $\mathrm{H} 2$. Or we can prove this theorem for betweenness functionals and obtain in part $2, V(a, 1)>V\left(a-\bar{L}, \frac{1}{2} ; a+\right.$

$\left.\bar{G}, \frac{1}{2}\right)$. Our main point is that the regularity imposed by the $\mathrm{H}$ assumptions on functionals enables us to weave together many pieces of reasonable risk aversion with respect to small lotteries into absurd risk aversion with respect to large lotteries.

\section{Appendix}

Proof of Theorem 1 We show first that for all $F, u(\cdot ; F)$ is concave. Let $\mathcal{I}$ be the supporting hyperplane of the indifference set of $V$ through $F$. Let $u(\cdot ; F)$ be the local utility determining this hyperplane (hence for all $H \in \mathcal{I}$, $\left.\int u(x ; F) d H(x)=\int u(x ; F) d F(x)\right)$. Suppose $u(\cdot ; F)$ is not concave, then there exist $z$ and $H$ such that $z=\mathrm{E}[H]$ and $\int u(x ; F) d H(x)>u(z ; F)$. By monotonicity and continuity, there exist $p$ and $z^{\prime}$ such that $\left(z, p ; z^{\prime}, 1-p\right) \in \mathcal{I}$, hence $H^{\prime}:=\left(H, p ; z^{\prime}, 1-p\right)$ is above $\mathcal{I}$ with respect to the expected utility functional with the vNM utility function $u(\cdot ; F)$. All lines in $\mathcal{I}$ are tangent at $F$ to the indifference curve of $V$ through $F$, hence, by assumption, $\mathcal{I}$ is the hyperplane that contains all the lines that are tangent at $F$ to that indifference set. As $H^{\prime} \notin \mathcal{I}$, the line through $F$ and $H^{\prime}$ is not in $\mathcal{I}$, hence it cannot be tangent at $F$ to the indifference set of $V$ though $F$. Therefore there exists $\alpha \in(0,1)$ such that $V\left(F, \alpha ; H^{\prime}, 1-\alpha\right)>V(F) \geqslant V\left(F, \alpha ;\left(z, p ; z^{\prime}, 1-p\right), 1-\alpha\right)$ (the second inequality follows by quasi concavity). This is a violation of risk aversion, as the first distribution is a mean preserving spread of the third.

CAse 1: Assume $\mathrm{H} 1$ and let $F_{b}$ be the distribution of $\left(b-\ell, \frac{1}{2} ; b+g, \frac{1}{2}\right)$. Using quasi-concavity and continuity, the preference $V(b, 1)>V\left(F_{b}\right)$ implies 
that $u\left(b ; F_{b}\right)>\frac{1}{2} u\left(b-\ell ; F_{b}\right)+\frac{1}{2} u\left(b+g ; F_{b}\right)$. By H1,

$$
u\left(x ; F_{b}\right)>\frac{1}{2} u\left(x-\ell ; F_{b}\right)+\frac{1}{2} u\left(x+g ; F_{b}\right)
$$

for all $x \leqslant b$. Taking $b-\ell$ to be the wealth level, Table 2 implies

$$
u\left(b-\ell ; F_{b}\right)>\varepsilon u\left(b-\ell-L ; F_{b}\right)+(1-\varepsilon) u\left(b-\ell+G ; F_{b}\right)
$$

Hence,

$$
\begin{aligned}
\int u\left(x ; F_{b}\right) d F_{b}(x) & =\frac{1}{2} u\left(b-\ell ; F_{b}\right)+\frac{1}{2} u\left(b+g ; F_{b}\right) \\
& >u\left(b-\ell ; F_{b}\right) \\
& >\varepsilon u\left(b-\ell-L ; F_{b}\right)+(1-\varepsilon) u\left(b-\ell+G ; F_{b}\right)
\end{aligned}
$$

which implies that, according to the local utility at $F_{b}, F_{b}$ is preferred to the lottery $(b-\ell-L, \varepsilon ; b-\ell+G, 1-\varepsilon)$. Hence, by quasi-concavity, $V\left(F_{b}\right)>$ $V(b-\ell-L, \varepsilon ; b-\ell+G, 1-\varepsilon)$ and, as $V(b, 1)>V\left(F_{b}\right)$,

$$
V(b, 1)>V(b-g-L, \varepsilon ; b-g+G, 1-\varepsilon)
$$

CAse 2: Assume H2 and consider $x \in[a, b-g]$. As in Case 1, the preference $V(x, 1)>V\left(x-\ell, \frac{1}{2} ; x+g, \frac{1}{2}\right)$ implies that at $F_{x}$, the distribution of $(x-$ $\left.\ell, \frac{1}{2} ; x+g, \frac{1}{2}\right)$,

$$
u\left(x ; F_{x}\right)>\frac{1}{2} u\left(x-\ell ; F_{x}\right)+\frac{1}{2} u\left(x+g ; F_{x}\right)
$$

Obviously, as $x \leqslant b-g, \delta_{b}$ dominates $F_{x}$ by first order stochastic dominance. Hence, by H2, $u\left(x ; \delta_{b}\right)>\frac{1}{2} u\left(x-\ell ; \delta_{b}\right)+\frac{1}{2} u\left(x+g ; \delta_{b}\right)$ for all $x \in[a, b-g]$. Table 2 now implies

$$
\begin{aligned}
& u\left(b ; \delta_{b}\right)>u\left(b-g ; \delta_{b}\right)> \\
& \quad \varepsilon u\left(b-g-L ; \delta_{b}\right)+(1-\varepsilon) u\left(b-g+G ; \delta_{b}\right)
\end{aligned}
$$

Hence, by quasi concavity,

$$
V(b, 1)>V(b-g-L, \varepsilon ; b-g+G, 1-\varepsilon)
$$

CASE 3: Assume $\neg$ H1. Similarly to Case 1, the preference $V(a, 1)>V(a-$ $\left.\ell, \frac{1}{2} ; a+g, \frac{1}{2}\right)$ implies that for $F_{a}$, the distribution of $\left(a-\ell, \frac{1}{2} ; a+g, \frac{1}{2}\right)$, the inequality

$$
u\left(a ; F_{a}\right)>\frac{1}{2} u\left(a-\ell ; F_{a}\right)+\frac{1}{2} u\left(a+g ; F_{a}\right)
$$


is satisfied and

$$
V(a, 1) \geqslant V\left(F_{a}\right)
$$

By $\neg \mathrm{H} 1$, the first inequality holds for all $x \geqslant a$ and, by Table 1 ,

$$
u\left(a ; F_{a}\right)>\frac{1}{2} u\left(a-\bar{L} ; F_{a}\right)+\frac{1}{2} u\left(a+\bar{G} ; F_{a}\right)
$$

Set $u\left(a ; F_{a}\right)=0$ and use the concavity of $u\left(\cdot ; F_{a}\right)$ to obtain

$$
u\left(a-\ell ; F_{a}\right) \geqslant \frac{\ell}{\bar{L}} u\left(a-\bar{L} ; F_{a}\right)
$$

Using the last two inequalities we obtain

$$
\begin{aligned}
& \int u\left(x ; F_{a}\right) d F_{a}(x)= \\
& \quad \frac{1}{2} u\left(a-\ell ; F_{a}\right)+\frac{1}{2} u\left(a+g ; F_{a}\right)> \\
& \quad \frac{1}{2} u\left(a-\ell ; F_{a}\right)+\frac{1}{2} u\left(a ; F_{a}\right)> \\
& \quad \frac{\ell}{2 \bar{L}} u\left(a-\bar{L} ; F_{a}\right)+\frac{1}{2}\left[\frac{1}{2} u\left(a-\bar{L} ; F_{a}\right)+\frac{1}{2} u\left(a+\bar{G} ; F_{a}\right)\right]> \\
& \quad \frac{3}{4} u\left(a-\bar{L} ; F_{a}\right)+\frac{1}{4} u\left(a+\bar{G} ; F_{a}\right)
\end{aligned}
$$

where the last inequality holds since $\frac{\ell}{2 \bar{L}}<\frac{1}{2}$ and $u\left(a-\bar{L} ; F_{a}\right)<0$. Finally, eq. (13) and quasi concavity imply

$$
V(a, 1) \geqslant V\left(F_{a}\right)>V\left(a-\bar{L}, \frac{3}{4} ; a+\bar{G}, \frac{1}{4}\right)
$$

CAse 4: We assume here that $\bar{L}>4 \ell$. Assume $\neg \mathrm{H} 2$ and obtain, similarly to Case 2 , that $u\left(x ; \delta_{a}\right)>\frac{1}{2} u\left(x-\ell ; \delta_{a}\right)+\frac{1}{2} u\left(x+g ; \delta_{a}\right)$ for all $x \in[a+\ell, b]$. Now, by Table 1 ,

$$
u\left(a+\ell ; \delta_{a}\right)>\frac{1}{2} u\left(a+\ell-\bar{L} ; \delta_{a}\right)+\frac{1}{2} u\left(a+\ell+\bar{G} ; \delta_{a}\right)
$$

Set $u\left(a+\ell ; \delta_{a}\right)=0$ and use the concavity of $u\left(\cdot ; \delta_{a}\right)$ to obtain

$$
u\left(a ; \delta_{a}\right) \geqslant \frac{\ell}{L} u\left(a+\ell-\bar{L} ; \delta_{a}\right)
$$

Summing the last two inequalities yields (recall that $u\left(a+\ell ; \delta_{a}\right)=0$ )

$$
\begin{aligned}
u\left(a ; \delta_{a}\right) & >\left(\frac{1}{2}+\frac{\ell}{L}\right) u\left(a+\ell-\bar{L} ; \delta_{a}\right)+\frac{1}{2} u\left(a+\ell+\bar{G} ; \delta_{a}\right) \\
& >\left(\frac{1}{2}+\frac{\ell}{L}\right) u\left(a+\ell-\bar{L} ; \delta_{a}\right)+\left(\frac{1}{2}-\frac{\ell}{L}\right) u\left(a+\ell+\bar{G} ; \delta_{a}\right) \\
& >\frac{3}{4} u\left(a+\ell-\bar{L} ; \delta_{a}\right)+\frac{1}{4} u\left(a+\ell+\bar{G} ; \delta_{a}\right)
\end{aligned}
$$


Where the last inequality holds since $\frac{\ell}{L}<\frac{1}{4}$ and $u\left(a+\ell-\bar{L} ; \delta_{a}\right)<0$. Finally, quasi concavity and monotonicity imply

$$
\begin{aligned}
V(a, 1) & >V\left(a+\ell-\bar{L}, \frac{3}{4} ; a+\ell+\bar{G}, \frac{1}{4}\right) \\
& >V\left(a-\bar{L}, \frac{3}{4} ; a+\bar{G}, \frac{1}{4}\right)
\end{aligned}
$$

Proof of Lemma 1 Suppose $u(\cdot ; F)$ is not concave. Then there exist $H^{*}$ and $H$ such that $H$ is a mean preserving spread of $H^{*}$, but $\int u(x ; F) d H^{*}(x)<$ $\int u(x ; F) d H(x)$. For every $\varepsilon,(1-\varepsilon) F+\varepsilon H$ is a mean preserving spread of $(1-\varepsilon) F+\varepsilon H^{*}$, hence, by risk aversion, $V((1-\varepsilon) F+\varepsilon H) \leqslant V\left((1-\varepsilon) F+\varepsilon H^{*}\right)$. As this inequality holds for all $\varepsilon$, it follows that $\left.\frac{\partial}{\partial \varepsilon} V((1-\varepsilon) F+\varepsilon H)\right|_{\varepsilon=0}$ $\leqslant\left.\frac{\partial}{\partial \varepsilon} V\left((1-\varepsilon) F+\varepsilon H^{*}\right)\right|_{\varepsilon=0}$. Hence, by equation $(10), \int u(x ; F) d H(x) \leqslant$ $\int u(x ; F) d H^{*}(x)$, a contradiction.

Proof of Theorem 2 Let $(\ell, g, L, G, \varepsilon)$ be a lower calibration quintuple with $\varepsilon<\hat{\varepsilon}$ and $G$ as is defined in eq. (11) (the existence of such a quintuple follows by eq. (9)).

Assume H2 and consider $x \in[a, b-g]$. As $V(x, 1)>V\left(x-\ell, \frac{1}{2} ; x+g, \frac{1}{2}\right)$, it follows by Gâteaux differentiability that there are $p$ and $\zeta>0$ such that for all $p^{\prime} \in(p, p+\zeta)$

$$
V\left(x, 1-p ; x-\ell, \frac{p}{2} ; x+g, \frac{p}{2}\right)>V\left(x, 1-p^{\prime} ; x-\ell, \frac{p^{\prime}}{2} ; x+g, \frac{p^{\prime}}{2}\right)
$$

Denote by $H_{x}$ the distribution of $\left(x, 1-p ; x-\ell, \frac{p}{2} ; x+g, \frac{p}{2}\right)$. By Gâteaux differentiability we obtain that for a sufficiently close $p^{\prime}, p^{\prime \prime} \in[p, p+\zeta], p^{\prime}<p^{\prime \prime}$,

$$
\begin{aligned}
& \left(1-p^{\prime}\right) u\left(x ; H_{x}\right)+\frac{p^{\prime}}{2} u\left(x-\ell ; H_{x}\right)+\frac{p^{\prime}}{2} u\left(x+g ; H_{x}\right)> \\
& \quad\left(1-p^{\prime \prime}\right) u\left(x ; H_{x}\right)+\frac{p^{\prime \prime}}{2} u\left(x-\ell ; H_{x}\right)+\frac{p^{\prime \prime}}{2} u\left(x+g ; H_{x}\right)
\end{aligned}
$$

Hence

$$
u\left(x ; H_{x}\right)>\frac{1}{2} u\left(x-\ell ; H_{x}\right)+\frac{1}{2} u\left(x+g ; H_{x}\right)
$$

Obviously, $\delta_{b}$ dominates $H_{x}$ by first order stochastic dominance whenever $b>x+g$. Hence, by H2, $u\left(x ; \delta_{b}\right)>\frac{1}{2} u\left(x-\ell ; \delta_{b}\right)+\frac{1}{2} u\left(x+g ; \delta_{b}\right)$ for all $x \in[a, b-g]$. As before, since local utilities are concave (Lemma 1), Table 2 now implies

$$
\begin{aligned}
& u\left(b ; \delta_{b}\right)>u\left(b-g ; \delta_{b}\right)> \\
& \quad \varepsilon u\left(b-g-L ; \delta_{b}\right)+(1-\varepsilon) u\left(b-g+G ; \delta_{b}\right)
\end{aligned}
$$


By Gâteaux differentiability, this implies that for sufficiently small $\mu$, the decision maker with wealth level $b$ prefers not to participate in the lottery $(b-g-L, \mu \varepsilon ; b, 1-\mu ; b-g+G, \mu(1-\varepsilon))$. We now show that $\mu=1$, which is the claim of the theorem.

Let $\bar{\mu}=\max \{\mu: V(b, 1) \geqslant V(b-g-L, \mu \varepsilon ; b, 1-\mu ; b-g+G, \mu(1-$ $\varepsilon))\}$ and suppose that $\bar{\mu}<1$. Denote by $\bar{F}$ the distribution of the lottery $(b-g-L, \bar{\mu} \varepsilon ; b, 1-\bar{\mu} ; b-g+G, \bar{\mu}(1-\varepsilon))$. We want to show that for all $x \in[b-g-L, b-g]$,

$$
u(x ; \bar{F})>\frac{1}{2} u(x-\ell ; \bar{F})+\frac{1}{2} u(x+g ; \bar{F})
$$

We defined $a=b-g-L$, therefore, as $\bar{\mu} \varepsilon<\hat{\varepsilon}, V\left(\hat{X}_{x}\right)>V\left(\tilde{X}_{x}\right)$, where $\hat{X}_{x}=(b-g-L, \bar{\mu} \varepsilon ; x, 1-\bar{\mu} \varepsilon)$ and $\tilde{X}_{x}=\left(b-g-L, \bar{\mu} \varepsilon ; x-\ell, \frac{1-\bar{\mu} \varepsilon}{2} ; x+g, \frac{1-\bar{\mu} \varepsilon}{2}\right)$. Let $\hat{F}_{x}$ and $\tilde{F}_{x}$ denote the distributions of $\hat{X}_{x}$ and $\tilde{X}_{x}$, respectively. Similarly to the derivation of eq. (16), it follows by Gâteaux differentiability that there exists $F$ in the line segment connecting $\hat{F}_{x}$ and $\tilde{F}_{x}$ for which

$$
u(x ; F)>\frac{1}{2} u(x-\ell ; F)+\frac{1}{2} u(x+g ; F)
$$

As $\bar{F}$ dominates both $\hat{F}_{x}$ and $\tilde{F}_{x}$ by first order stochastic dominance it dominates $F$ as well and eq. (18) follows by $\mathrm{H} 2$.

Similarly to the derivation of eq. (17), the local utility at $\bar{F}$ satisfies

$$
u(b ; \bar{F})>\varepsilon u(b-g-L ; \bar{F})+(1-\varepsilon) u(b-g+G ; \bar{F})
$$

Let $H$ denote the cumulative distribution function of $(b-g-L, \varepsilon ; b-g+$ $G, 1-\varepsilon)$. Then, by Gâteaux differentiability and eq. (19),

$$
\begin{aligned}
& \frac{\partial}{\partial t} V((1-t) \bar{F}+t H)= \\
& \quad(1-\bar{\mu})[\varepsilon u(b-g-L ; \bar{F})+(1-\varepsilon) u(b-g+G ; \bar{F})-u(b ; \bar{F})]<0
\end{aligned}
$$

But this means that $\exists \mu \in(\bar{\mu}, 1)$ such that $V(b-g-L, \mu \varepsilon ; b, 1-\mu ; b-g+$ $G, \mu(1-\varepsilon))<V(b-g-L, \bar{\mu} \varepsilon ; b, 1-\bar{\mu} ; b-g+G, \bar{\mu}(1-\varepsilon)) \leqslant V(b, 1) ;$ a contradiction. Hence $\bar{\mu}=1$ and

$$
V(b, 1) \geqslant V(b-g-L, \varepsilon ; b-g+G, 1-\varepsilon)
$$




\section{References}

[1] Allais, M., 1953. "Le comportement de l'homme rationnel devant le risque: Critique des postulates et axiomes de l'ecole Americaine," Econometrica 21:503-546.

[2] Battalio, R.C., J.H. Kagel, and K. Jiranyakul, 1990. "Testing between alternative models of choice under uncertainty: Some initial results," Journal of Risk and Uncertainty 3:25-50.

[3] Chew, S.H., 1983. "A generalization of the quasilinear mean with applications to the measurement of income inequality and decision theory resolving the Allais paradox," Econometrica 51:1065-1092.

[4] Chew, S.H., 1989. "Axiomatic utilty theories with the betweenness property," Annals of Operations Research 19:273-298.

[5] Chew, S.H., L.G. Epstein, and U. Segal, 1991. "Mixture symmetry and quadratic utility," Econometrica 59:139-163.

[6] Chew, S.H., E. Karni, and Z. Safra, 1987. "Risk aversion in the theory of expected utility with rank-dependent probabilities," Journal of Economic Theory 42:370-381.

[7] Conlisk, J., 1989. "Three variants on the Allais example," American Economic Review 79:392-407.

[8] Dekel, E., 1986. "An axiomatic characterization of preferences under uncertainty: Weakening the independence axiom," Journal of Economic Theory 40:304-318.

[9] Epstein, L.G., 1992. "Behavior under risk: Recent developments in theory and applications," in J.J. Laffont (ed.): Advances in Economic Theory, vol. II. Cambridge: Cambridge University Press, pp. 1-63.

[10] Gul, F., 1991. "A theory of disappointment aversion," Econometrica 59:667-686.

[11] Hansson, B., 1988. "Risk aversion as a problem of conjoint measurment," in Decision, Probability, and Utility, ed. by P. Gardenfors and N.-E. Sahlin. Cambridge: Cambridge University Press, 136-158. 
[12] LeRoy, S., 2003. "Expected utility: A defence," Ecomomics Bulletin $7(7): 1-3$.

[13] Machina, M.J., 1982. "'Expected utility' analysis without the independence axiom," Econometrica 50:277-323.

[14] Machina, M.J., 1987. "Choice under uncertainty: Problems solved and unsolved," Journal of Economic Perspectives 1:121-154.

[15] Palacios-Huerta, I. and R. Serrano, 2006. "Rejecting Small Gambles Under Expected Utility," Economics Letters 91:250-259.

[16] Quiggin, J., 1982. "A theory of anticipated utility," Journal of Economic Behavior and Organization 3:323-343.

[17] Rabin, M., 2000. "Risk aversion and expected utility theory: A calibration result," Econometrica 68:1281-1292.

[18] Safra, Z. and U. Segal, 2006. "Are universal preferences possible? Calibration results for non-expected utility theories," mimeo.

[19] Segal, U. and A. Spivak, 1990. "First order versus second order risk aversion," Journal of Economic Theory 51:111-125.

[20] Segal, U. and A. Spivak, 1997. "First-order risk aversion and nondifferentiability," Economic Theory, 9:179-183.

[21] Starmer, C., 2000. "Developments in non-expected utility theory: The hunt for a descriptive theory of choice under risk," Journal of Economic Literature 38:332-382.

[22] Yaari, M.E., 1987. "The dual theory of choice under risk," Econometrica 55:95-115.

[23] Zeidler, E., 1985. Nonlinear Functional Analysis and its Applications, vol. III. New York: Springer. 